\title{
Treatment of auditory processing in noise in individuals with mild aphasia: pilot study
}

\author{
Anastasia M. Raymer ${ }^{1}$, Hilary M. Sandberg ${ }^{1}$, Kathryn S. Schwartz ${ }^{1}$ Ginger S. Watson², Stacie I. Ringleb \\ 'Old Dominion University, Norfolk, VA; ${ }^{2}$ University of Virginia, Charlottesville, VA, USA
}

Purpose: Listening in noise challenges listeners with auditory comprehension impairments in aphasia. We examined the effects of Trivia Game, a computerized program with questions spoken in increasing levels of background noise with success in the game.

Methods: We piloted Trivia Game in four individuals with chronic aphasia and mild auditory comprehension impairments. Participants played Trivia Game for 12 twenty-minute sessions. In addition to the Western Aphasia Battery (WAB), we measured outcomes on Quick Speech in Noise (QSIN), a sentence repetition test, administered in auditory (AUD) and auditory+visual (AV) conditions as signal-to-noise ratio varied from 25 to $0 \mathrm{~dB}$.

Results: All four participants showed progress within the game in the noise level attained. Increases in repetition accuracy were seen in two participants for the QSIN AUD condition (average of 5.5 words), and in three participants for QSIN AV (average of 16.5 words). One individual increased performance on the WAB.

Conclusions: Use of Trivia Game led to improved auditory processing abilities in all four individuals with aphasia. Greater gains noted in the AV condition over AUD suggest that Trivia Game may facilitate speech-reading skills to support comprehension of speech in situations with background noise.

Keywords: Aphasia, Auditory comprehension, Treatment, Noise

\section{INTRODUCTION}

Auditory comprehension impairments are a common manifestation of aphasia in individuals who incur left cerebral damage. Even individuals with mild aphasia often report difficulties with auditory processing in daily activities, such as listening to the television or movies. These difficulties tend to be exacerbated in deleterious listening conditions, such as noisy medical facilities where these individuals participate in rehabilitation, or in noisy restaurants once they resume daily activities.

A large literature has examined factors that impact auditory comprehension impairments in individuals with aphasia, such as linguistic components [1] and processing conditions (e.g., rate of speech, time to respond) [2]. One area with more limited systematic study is the impact of degraded listening conditions for auditory processing abilities in individuals with aphasia. In the distant past, Basili et al. [3] reported that individuals with aphasia had inordinate difficulty listening to speech when presented in the presence of either white noise or speech noise as compared to healthy control subjects. More recent studies have reported the detrimental effects of white noise or MRI

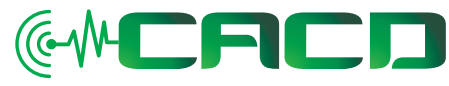

Received: July 13, 2019

Revision: July 25, 2019

Accepted: August 15, 2019

Correspondence:

Anastasia M. Raymer

Old Dominion University, 241 Child Study Center, 4501 Hampton Blvd., Norfolk, VA, USA

Tel: +7576834522

Fax: +7576835593

E-mail: sraymer@odu.edu

(C) 2019 The Korean Association of SpeechLanguage Pathologists

This is an Open Access article distributed under the terms of the Creative Commons Attribution NonCommercial License (http://creativecommons.org/ licenses/by-nc/4.0/) which permits unrestricted noncommercial use, distribution, and reproduction in any medium, provided the original work is properly cited. 
scanner noise on auditory processing in individuals with aphasia $[4,5]$. These studies focused largely on processing of only single words or syllables. Studies using sentence level text in noise are less common.

In degraded listening conditions, as in listening to speech in background noise, the visual modality becomes especially important to facilitate auditory recognition and comprehension [6]. Some researchers suggest that providing visual information may in fact be distracting and detrimental to auditory comprehension in individuals with aphasia [7]. Sandberg et al. [8] examined the influence that visual information provides for speech processing in noise in individuals with aphasia. They compared a standardized auditory sentence repetition test, Quick Speech in Noise (QSIN) [9] to a modified version presented with auditory+visual face information. As noise levels increased, the visual modality provided significant support for auditory processing for individuals with aphasia, as in healthy controls. However, at the most difficult listening level (signal-to-noise ratio [SNR] of $0 \mathrm{~dB}$ ), individuals with aphasia experienced less gain than expected. This suggests that individuals with aphasia may need additional intervention to take advantage of visual speech information in listening conditions involving background noise.

\section{Treatment for auditory comprehension in aphasia}

A limited number of studies have examined treatments for auditory comprehension impairments in aphasia. Most of those treatments have studied manipulations of linguistic conditions such as syntactic [10], semantic [11,12], or phonologic processing [13]. Less is known about interventions that alter and challenge listening conditions during auditory processing.

Clinicians are turning more frequently to computerized training tasks to address language impairments in aphasia. This is especially relevant when training language comprehension skills [14] where an auditory stimulus can be presented followed by a patient response in the form of a simple computer keystroke. Of the few studies that have examined computerized training of auditory comprehension in aphasia, most have incorporated isolated lexical tasks [12]. Less is known about training programs that simulate real-life listening situations, including noisy listening conditions, for individuals with aphasia.

\section{Trivia Game}

Restaurants provide one of the most challenging environ- ments for all listeners due to increased levels of background noise [15]. Therefore, we developed a computerized Trivia Game for rehabilitation of listening in noise that simulates a noisy restaurant. Trivia questions are presented aloud by a "waiter" viewed on a video monitor as background noise simulates a noisy restaurant with talking and clanging glass and utensils (Figure 1). The correct answer along with five foils are printed on the video screen for selection on a keyboard. Players receive points for timely selection of the correct answer. Clues are provided to rule out wrong answers and to gain additional points in the game. The level of background noise changes depending on the accuracy of responses in the game. The 23 SNR levels in the game range from $27 \mathrm{~dB}$ to $-39 \mathrm{~dB}$. A series of four of five correct responses leads to an increase in the level of background noise, whereas a series of two of five incorrect responses leads to a decrease in the level of background noise. The game allows the player to choose categories such as foods, animals, movies, and sports, with 40-50 questions per category as a strategy to enhance top-down processing that is an important element of auditory processing in difficult listening conditions [16]. To further support top-down processing, players can see the speaker on the video monitor.

Trivia Game was originally tested in a group of healthy college-aged listeners [17]. Following 12 sessions of playing the game for 20 minutes, participants improved in the SNR level achieved within the game, increased scores on Quick Speech in Noise (QSIN) [9], an audiovisual listening in noise test, and reported functional benefits of the game in the Speech, Spatial and Qualities of Hearing Scale (SSQ-B) [18]. In a preliminary study, adults with hearing loss also showed benefits on standardized testing after playing Trivia Game [19].

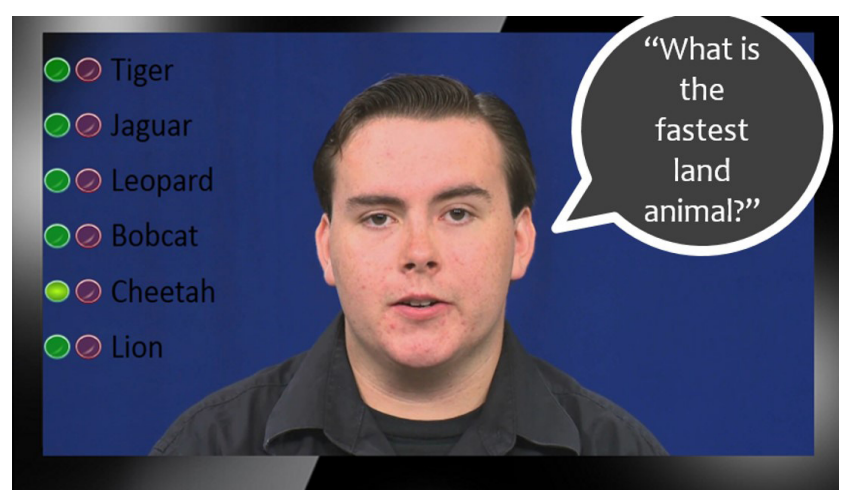

Figure 1. Example of speaker presenting trivia question along with written choices. 


\section{Purpose}

In the current project, we piloted the use of Trivia Game in four participants with stroke-induced aphasia and mild auditory comprehension impairments. We aimed to determine whether individuals with aphasia could benefit from playing Trivia Game by showing progress in noise level attained within the game and improvements in auditory processing scores for auditory and auditory-visual presentations on auditory processing tests assessing listening in noise and aphasia. This feasibility study was conducted to inform our development of Trivia Game for individuals with auditory processing impairments and to accrue pilot data in individuals with aphasia.

\section{METHODS}

\section{Participants}

The four right-handed men (48-65 years old) had experienced aphasia following a left hemisphere stroke that occurred more than one year earlier (range 13-57 months post onset). All completed more than 12 years of education and spoke English as their primary language. In audiometric testing, three participants (Aph1-3) had hearing thresholds $\leq 25 \mathrm{~dB}$ HL for pure-tone frequencies $250-8,000 \mathrm{~Hz}$, bilaterally; Aph4 had a unilateral moderate high frequency hearing loss. To characterize aphasia profiles, participants were administered the Western Aphasia Battery-Revised (WAB-R) [20], a standardized aphasia test with subtests assessing fluency of verbal production, auditory comprehension, repetition, and word retrieval. WAB-R results showed that Aph1 and Aph4 had fluent forms of aphasia (conduction aphasia and anomic aphasia, respectively) and Aph2 and Aph3 had nonfluent aphasia (both Broca's aphasia). All were able to repeat simple sentences in the WAB-R Repetition subtest despite occasional phonologic or articulatory errors. All had relatively mild auditory comprehension impairments as demonstrated by scores ranging from 8.6-9.7 on the WAB Auditory Comprehension subtest (maximum score $=10$ ). Participant demographic characteristics are detailed in Table 1. All participants provided written informed consent to take part in this research project.

\section{Trivia Game play}

To address their mild auditory comprehension difficulties, the four participants with aphasia played the computerized Trivia Game described earlier. In the first laboratory training session, participants were given verbal and written instruction on how to use Trivia Game. They were instructed to set the volume at a comfortable listening level and to keep it at that level for each training session. They then played the game independently at home in a quiet location on a laptop provided for their use. Participants played the game for 20-minute sessions four times per week for three weeks for a total of 12 sessions. In each session they played a different trivia category as instructed on a schedule to avoid repeated exposure to the same trivia questions over time. Game results were noted by each participant on a written log, which allowed for tracking of the time spent playing and noise level attained for each session of game play over the twelve study sessions. A researcher checked in with the participants weekly to address any questions or concerns.

\section{Outcome measures}

The primary outcome measure administered pre- and postgame play was Quick Speech in Noise (QSIN) [9], a sentence repetition test in the presence of background speech noise (four-talker babble). QSIN sentences come from a standard set of sentences that were devised so as not to be predictable from the sentence context (e.g., A white silk jacket goes with

Table 1. Participant demographic characteristics and aphasia test results

\begin{tabular}{lcccc}
\hline Participant & APH 1 & APH 2 & APH 3 & APH 4 \\
\hline Gender & M & M & M & M \\
Age (year) & 48 & 65 & 58 & 64 \\
Education (year) & 20 & 16 & 12 & 14 \\
Time Post CVA (month) & 13 & 57 & 52 & 14 \\
Hearing acuity & WNL & WNL & WNL & Unilateral Left \\
& & & & High Freq. \\
& & & & Hearing Loss \\
Aphasia type & Conduction & Broca's & Broca's & Anomic \\
Pre-test WAB-R & & & & \\
A0 (Max=100) & 82.7 & 72.4 & 62.6 & 90.8 \\
Auditory Comp. & 9.05 & 8.6 & 9.05 & 9.7 \\
Repetition & 5.4 & 7.5 & 6.2 & 9.2 \\
Naming & 8.9 & 7.1 & 7.5 & 8.5 \\
Spont. Speech & 18 & 13 & 8 & 18 \\
Post-test WAB-R Change & & & & \\
A0 & -3.4 & 2.2 & 3.9 & 5.4 \\
Auditory Comp. & -0.7 & 0.9 & -0.5 & 0.2 \\
Repetition & 0.2 & -0.5 & 0 & 0.4 \\
Naming & -0.2 & -0.2 & 0.5 & 0.1 \\
Spont. Speech & -1.0 & 0 & 1.0 & 2.0 \\
\hline
\end{tabular}


any shoes; The child crawled into the dense grass). QSIN was administered in the standard auditory (AUD) condition and in an experimental auditory+visual face (AV) condition, with order of conditions counterbalanced across participants. In the AUD condition, the participants heard sentences spoken by a female voice through headphones at $70 \mathrm{~dB} \mathrm{HL}$ as a $\mathrm{CD}$ player routed sentences through a GSI-61 audiometer to TDH-50 headphones. Signal-to-noise ratio (SNR) varied from +25 to $0 \mathrm{~dB}$ in $5 \mathrm{~dB}$ decrements for each sentence in six sentence blocks. In the AV condition, the participants could also see the female speaker on a video monitor as they listened to sentences with the headphones. A practice list of six sentences was used in each condition to familiarize each participant with the test requirements. Participants then repeated eight blocks of six sentences per condition. The examiner marked each sentence during testing for the presence of five key words, primarily nouns, verbs, adjectives, or prepositions (underlined words in the earlier examples). Spoken responses also were audio-recorded to assure reliability of scoring. We calculated the number of key words repeated correctly across six SNR levels for AUD and AV conditions ( $\max$ score $=40$ per SNR, total max score per condition $=240$ ). Distortions due to apraxia of speech or simple phonemic paraphasias were ac-

Table 2. QuickSIN scores at pre-training and post-training across SNR levels (max $=40$ per $\mathrm{dB}$ level)

\begin{tabular}{|c|c|c|c|c|c|c|c|c|}
\hline \multirow{2}{*}{$\begin{array}{l}\text { Pre-Training } \\
\text { SNR Level }\end{array}$} & \multicolumn{2}{|c|}{ APH1 } & \multicolumn{2}{|c|}{ APH2 } & \multicolumn{2}{|c|}{ APH3 } & \multicolumn{2}{|c|}{ APH4 } \\
\hline & Aud & $A+V$ & Aud & $A+V$ & Aud & $A+V$ & Aud & $\mathrm{A}+\mathrm{V}$ \\
\hline $25 \mathrm{~dB}$ & 3 & 3 & 34 & 33 & 34 & 28 & 40 & 40 \\
\hline $20 \mathrm{~dB}$ & 5 & 4 & 33 & 31 & 26 & 25 & 39 & 40 \\
\hline $15 \mathrm{~dB}$ & 5 & 4 & 27 & 31 & 27 & 29 & 40 & 40 \\
\hline $10 \mathrm{~dB}$ & 2 & 5 & 29 & 25 & 29 & 24 & 37 & 40 \\
\hline $5 \mathrm{~dB}$ & 2 & 3 & 5 & 21 & 15 & 15 & 32 & 39 \\
\hline $0 \mathrm{~dB}$ & 0 & 1 & 0 & 0 & 0 & 2 & 0 & 2 \\
\hline $\begin{array}{l}\text { Total score } \\
\text { (max=240) }\end{array}$ & 17 & 20 & 128 & 141 & 131 & 123 & 188 & 201 \\
\hline Post-Training & Aud & $A+V$ & Aud & $A+V$ & Aud & $A+V$ & Aud & $A+V$ \\
\hline $25 \mathrm{~dB}$ & 0 & 7 & 34 & 38 & 29 & 24 & 40 & 40 \\
\hline $20 \mathrm{~dB}$ & 7 & 10 & 37 & 34 & 27 & 27 & 40 & 40 \\
\hline $15 \mathrm{~dB}$ & 2 & 7 & 31 & 37 & 29 & 27 & 40 & 40 \\
\hline $10 \mathrm{~dB}$ & 1 & 7 & 27 & 36 & 25 & 24 & 38 & 37 \\
\hline $5 \mathrm{~dB}$ & 1 & 1 & 28 & 32 & 13 & 24 & 36 & 36 \\
\hline $0 \mathrm{~dB}$ & 0 & 4 & 0 & 8 & 1 & 9 & 0 & 2 \\
\hline $\begin{array}{l}\text { Total score } \\
\qquad(\max =240)\end{array}$ & 11 & 36 & 157 & 185 & 124 & 135 & 194 & 195 \\
\hline
\end{tabular}

cepted as correct responses. QSIN was administered once at baseline and again immediately post-completion of twelve sessions of Trivia Game play. Participants also completed the WAB-R post-training to document any generalized language changes associated with game play.

\section{RESULTS}

\section{Training logs}

According to daily training logs, all four participants with aphasia played 12 sessions of Trivia Game as planned. Logs indicated that after 12 sessions, Aphl stayed at the easiest noise level ( $\mathrm{SNR}=27 \mathrm{~dB}$ ), as his response accuracy decreased each time the noise level increased. The others three participants with aphasia played for 12 sessions at increasingly harder SNR levels over time (greater background noise levels) with Aph2 and Aph4 ending at level 12 (SNR =-6 dB), and Aph3 ending at level $14(\mathrm{SNR}=-12 \mathrm{~dB})$.

\section{QSIN}

Table 2 displays participant results for the QSIN for AUD and AV conditions across SNR levels. In the pre-treatment QSIN, Aph1, with conduction aphasia, accurately repeated only $37 / 480$ words in the two conditions, with only a +3 advantage for the AV condition over the AUD condition and little difference across SNR levels. At pre-treatment, Aph2, who repeated 269/480 words, and Aph4 who repeated 389/480 words both showed $\mathrm{a}+13$ word advantage for the AV condition over the AUD condition. In contrast, Aph3, who correctly repeated $254 / 480$ words overall at pre-treatment, produced +8 more words correctly in the AUD condition than the AV condition. Aph2, Aph3, and Aph4 showed the expected drop-off in performance at difficult SNR levels ( $5 \mathrm{~dB}$ and $0 \mathrm{~dB}$ ).

Post-training individual scores are presented in Table 2.

Table 3. Change in individual QSIN scores for auditory (AUD) and auditory+visual (AV) conditions from pre- to post-treatment with Trivia Game

\begin{tabular}{|c|c|c|c|c|c|c|c|c|}
\hline \multirow{2}{*}{$\begin{array}{l}\text { Gains } \\
\text { SNR }\end{array}$} & \multicolumn{2}{|c|}{ APH1 } & \multicolumn{2}{|c|}{$\mathrm{APH} 2$} & \multicolumn{2}{|c|}{ APH 3} & \multicolumn{2}{|c|}{ APH4 } \\
\hline & AUD & $A+V$ & AUD & $A+V$ & AUD & $A+V$ & AUD & $A+V$ \\
\hline $25 \mathrm{~dB}$ & -3 & +4 & 0 & +5 & -5 & -4 & 0 & 0 \\
\hline $20 \mathrm{~dB}$ & +2 & +6 & +4 & +3 & +1 & +2 & +1 & 0 \\
\hline $15 \mathrm{~dB}$ & -3 & +3 & +4 & +6 & +2 & -2 & 0 & 0 \\
\hline $10 \mathrm{~dB}$ & -1 & +2 & -2 & +11 & -4 & 0 & +1 & -3 \\
\hline $5 \mathrm{~dB}$ & -1 & -2 & +23 & +11 & -2 & +9 & +4 & -3 \\
\hline $0 \mathrm{~dB}$ & 0 & +3 & 0 & +8 & +1 & +7 & 0 & 0 \\
\hline
\end{tabular}




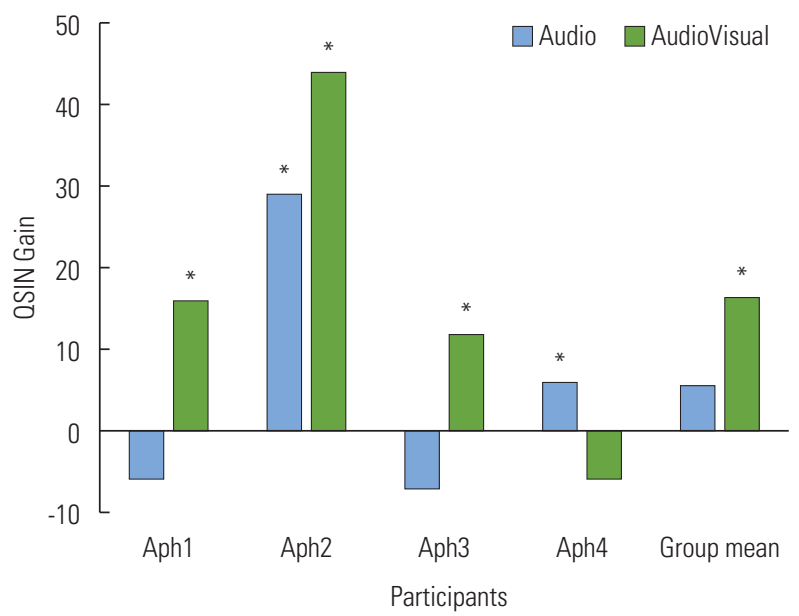

Figure 2. Gains in total score pre- versus post-treatment QSIN. ${ }^{*}>$ SEM (Audio=4.34; AudioVisual =5.52)

Gains for each participant comparing post-training to pretraining QSIN scores are shown in Table 3 and in Figure 2. Changes were compared to the standard error of measurement (SEM: $\mathrm{AUD}=4.34 ; \mathrm{AV}=5.52$ ) calculated on the basis of performance of healthy subjects administered QSIN on two occasions without exposure to the Trivia Game in our earlier study [17]. Meaningful gains were evident for two of four participants (Aph2 and 4) in the AUD condition (average gain = 5.5 words) and three of four (Aph1, 2 and 3) in the AV condition (average gain $=16.5$ words). Only Aph4, who performed near ceiling at baseline did not show AV condition gains. Greater gains were evident in the AV condition, despite the fact that it was the easier condition with higher scores at pretreatment.

Gains from pre- to post-treatment on the QSIN across SNR levels averaged for the group are shown in Figure 3. In the AUD condition, no clear pattern of change emerged in the 25 to $10 \mathrm{~dB}$ SNR levels. However, at $5 \mathrm{~dB}$ SNR, the group average gain of +6 words is influenced especially by Aph2 who made remarkable gains of +23 additional words repeated correctly following training. In the AV condition, increases were evident for the group at all SNR levels, with larger increases at the two most difficult SNR levels: +3.75 words at $5 \mathrm{~dB}$ and +4.5 words at $0 \mathrm{~dB}$, where signal and noise are at the same loudness level and visual information becomes crucial to interpret the signal.

\section{WAB-R}

Table 1 displays WAB- $\mathrm{R}$ change scores comparing pre- and post-treatment results. Only Aph4 increased greater than the SEM on the WAB for the overall Aphasia Quotient, as he dem-

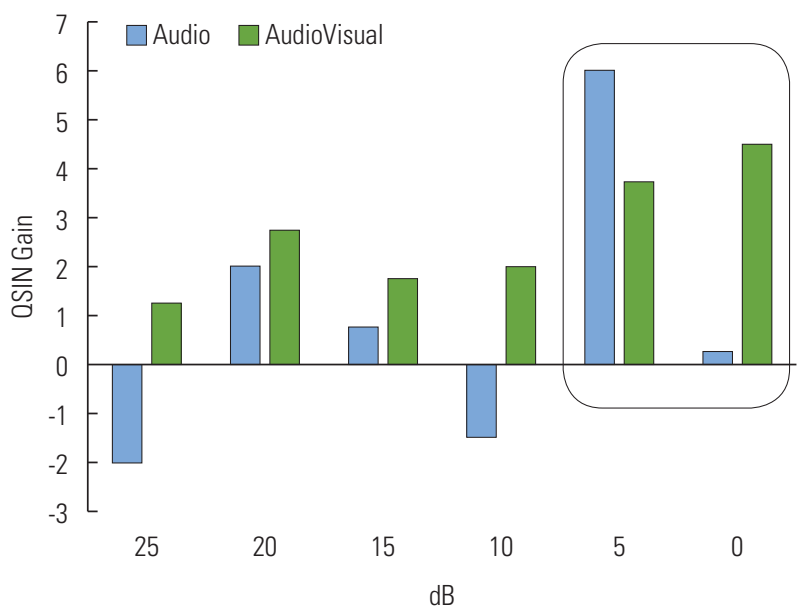

Figure 3. Average gains pre- versus post-treatment QSIN scores across SNR levels.

onstrated slight increases in each subtest. Interestingly, he was the individual with smallest gains on QSIN because of close to ceiling performance at pre-treatment.

\section{DISCUSSION}

Aphasia treatments for auditory processing in challenging listening conditions are limited in their availability. Therefore, we explored the effects of playing our newly developed computerized Trivia Game in a small group of participants with aphasia. Trivia Game simulates a noisy restaurant situation, thereby exposing participants to real-life sentence-level language processing. The game set-up facilitates top-down processing skills by presenting trivia questions within one semantic category and by providing visual face information as questions are presented.

After twelve sessions of playing Trivia Game, all four participants demonstrated changes in their auditory processing results. Within the game, three of four participants with aphasia played at increasingly more difficult SNR levels over time, reaching as low as -12 dB SNR. Despite their impressive progress, this level of game play was not comparable to the challenging levels attained by healthy young listeners in our earlier study who reached - 12 to -39 dB SNR after 12 training sessions [17]. Individuals with aphasia have limitations posed by the language impairments and may need more sessions than healthy individuals to reach the most difficult levels. Furthermore, individuals with aphasia may have cognitive and/or physical limitations that might have influenced their game play on the computer using a mouse to respond to trivia questions. 


\section{Standardized test findings}

The primary outcome measure used in this pilot study was QSIN, a standardized sentence repetition task that systematically varies SNR levels from easy to very difficult, where signal and noise are presented at the same noise level. While individuals with aphasia can have difficulty with sentence repetition tasks related to aphasic verbal production difficulties, repetition difficulty would be evident across all SNR levels, including the easiest levels. As results show in Table 2, Aphl had the most difficulty with QSIN across all SNR levels, demonstrating only a +3 advantage in the auditory-visual condition over auditory-only at baseline and minimal differences across SNR levels. In contrast, Aph2, Aph3, and Aph4 had much milder aphasic verbal production difficulties influencing their QSIN results at baseline, as indicated by their performance at the easiest SNR levels. QSIN performance dropped at the most difficult SNR levels for all three, suggesting the auditory contributions to their SNR results. At baseline, Aph2 and Aph4 showed +13 point advantages in the audiovisual condition relative to the audio-only condition. In contrast, Aph3 showed an +8 point advantage of audio-only over audiovisual at baseline. Our findings suggest that, whereas audio-visual information is beneficial for most individuals with aphasia, there can be some who find visual information deleterious to auditory processing, as contended by Youse et al. [7]. An intervention, such as Trivia Game, which facilitates practice with the use of visual information to support auditory processing would then be especially useful.

Although aphasic verbal production impairments may influence performance on QSIN, changes in performance across SNR levels after playing Trivia Game likely represent auditory processing contributions to the QSIN results. All four participants with aphasia showed increases beyond the measurement error in either the AUD or the AV condition following Trivia Game play. Aphl, with the most severe verbal production contributions to QSIN results, showed a +16 gain for the $\mathrm{A}+\mathrm{V} \mathrm{QSIN}$ condition following game play, suggesting considerable auditory processing improvements. Three of four individuals with aphasia had greater gains in the AV condition over the AUD condition post-training with Trivia Game. Aph2 and Aph3, in particular, demonstrated large gains at the most difficult listening levels (SNR $5 \mathrm{~dB}$ and $0 \mathrm{~dB}$ ) following game play. Only Aph4 who performed at ceiling levels in the QSIN $\mathrm{AV}$ condition at pre-treatment did not show meaningful change post-training. Yet Aph4 was the one participant who showed an increase on the WAB-R following training. This pattern of findings suggests that these four individuals benefited from playing Trivia Game. We propose that the game especially trained these individuals to focus on visual face information that was available as the waiter spoke the trivia questions as the auditory signal became more and more difficult to hear in the context of the restaurant background noise during game play.

\section{CONCLUSIONS}

In this case series exploratory study, we realize that we have limited demonstration of experimental control. Yet all four individuals were in chronic phases of aphasia recovery at $>12$ months post stroke onset. In addition, three individuals had no change on the WAB-R. Therefore, we temper our conclusions of positive gains following Trivia Game play in the context of this limitation. One other factor that may have affected the listeners with aphasia is that the speaker in Trivia Game was a man and the speaker in the QSIN is a woman. Yet despite this difference, considerable changes were evident on QSIN testing, suggesting that the individuals with aphasia truly benefited from Trivia Game play to generally improve their auditory-visual processing of spoken language.

This pilot study allowed us to learn about the potential use of Trivia Game with a neurologically-impaired group. In the original version of Trivia Game, players hear the trivia question followed by clues to rule out incorrect answers. This step proved to be confusing for some participants in our healthy listener study [17], and was especially true for participants with aphasia. We are using these results to reformat the game to incorporate positive clues to the correct answer. Despite these challenges, the participants with aphasia willingly played Trivia Game as instructed and appeared to benefit from the experience. Future research will explore the effects of playing this listening in noise Trivia Game in a large cohort of individuals with aphasia.

\section{REFERENCES}

1. Martin RC, Tan Y. Sentence comprehension deficits: Independence and interaction of syntax, semantics, and working memory. Hillis AE, editor. The Handbook of Adult Language Disorders, 2nd ed. New York, NY: Psychology Press; 2015. p.303.

2. Davis GA. Aphasia and Related Cognitive-Communicative Disorders. Boston, MA: Pearson; 2014.

3. Basili AG, Diggs C, Rao P. Auditory processing of brain-damaged adults under competitive listening conditions. Brain Lang. 1980;9: 
362-371.

4. Healy EW, Moser DC, Morrow-Odom KL, Hall DA, Fridriksson J. Speech perception in MRI scanner noise by persons with aphasia. J Speech, Lang, Hear Res. 2007;50:323-334.

5. Kittredge A, Davis L, Blumstein SE. Effects of nonlinguistic auditory variations on lexical processing in Broca's aphasia. Brain Lang. 2006;97:25-40.

6. Jesse E, Janse A. Working memory affects older adults' use of context in spoken-word recognition. Q J Exp Psych. 2014;67:18421862.

7. Youse KM, Cienkowski KM, Coelho CA. Auditory-visual speech perception in an adult with aphasia. Brain Inj. 2004;18:825-834.

8. Sandberg HM, Ringleb S, Watson G, Morrison J, Deutsch M, Raymer AM. Impact of listening in noise with visual information in aphasia. Poster presented at American Speech-LanguageHearing Association, San Diego, CA, 2011.

9. Killion M, Niquette P, Gudmundsend G, Revit L, Banerjee S. Development of a quick speech in noise test for measuring signal to noise ratio loss in normal-hearing and hearing-impaired listeners. J Acoust Soc Amer. 2004;116:2395-2405.

10. Kiran S, Caplan D, Sandberg C, Levy J, Berardino A, Ascenso E, et al. Development of a theoretically based treatment for sentence comprehension deficits in individuals with aphasia. Amer J of Speech Lang Path. 2012;21:S88-S102.

11. Morris J, Franklin S. Investigating the effect of a semantic therapy on comprehension in aphasia. Aphasiol. 2012;26:1461-1480.

12. Raymer AM, Kohen F, Saffell D. Computerized training for impair- ments of word comprehension and retrieval in aphasia. Aphasiol. 2006;20:257-268.

13. Tessier C, Weill-Chounlamountry A, Michelot N, Pradat-Diehl P. Rehabilitation of word deafness due to auditory analysis disorder. Brain Inj. 2007;21:1165-1174.

14. Des Roches CA, Kiran S. Technology-based rehabilitation to improve communication after acquired brain injury. Front Neurosci. 2017;11:382.

15. Anderson S, Kraus N. Sensory-cognitive interaction in the neural encoding of speech in noise: A review. J Amer Acad Audiol. 2010; 29:575-585.

16. Sweetow R, Palmer CV. Efficacy of individual auditory training in adults: A systematic review of the evidence. J Amer Acad Audiol. 2005;16:494-504.

17. Schwartz KS, Ringleb S, Raymer AM, Sandberg H, Watson G. Development of Trivia Game for speech understanding in background noise. Intl J Speech Lang Path. 2015;17:357-366.

18. Jensen N, Akeroyd M, Noble W, Naylor G. The Speech, Spatial and Qualities of Hearing Scale (SSQ) as a benefit measure. Paper presented at the Natl Center Rehab Aud Res (NCRAR) Conference, Portland, OR, 2009.

19. Schwartz K, Kellner A, Griffin S, Ringleb S, Watson G, Raymer A. A new treatment approach for speech understanding in noise. Poster presented at Speech Hearing Assn Virginia, Chantilly, VA, 2016.

20. Kertesz A. Western aphasia battery-revised. San Antonio: Psych Corp, 2007. 